the need for strong adhoc clinician support to deal with complex patients and dedicated time for ongoing professional development for the IBD nurses. High volume of phone calls resulting in chasing investigations, conveying results and liaising with administrative team on behalf of patients necessitate better education about the IBD advice line. $57 \%$ of the patients who called had recently had a clinic appointment or was due one; with very little training given on running telephone clinics, one wonders about the clinical effectiveness of telephone clinics and whether the clinicians are able to address all of patients' concerns during these appointments.

During the COVID-19 pandemic, IBD advice line has been a valuable resource to patients. From safety and cost effectiveness (IBD advice line cost - $£ 39 /$ call, A\&E attendance - $£ 148$ / visit) point of view, it continues to enhance patient experience.

\section{PTH-61 FAECAL ELASTASE MAY HAVE A ROLE IN THE INVESTIGATION OF IRON DEFICIENCY ANAEMIA}

Faisal Shaikh*, Sadaf Dar, Richard Shenderey, Susanna West, Lionel Gracey-Whitman, Ahmad Zakaria, Fahad Shaikh, Chris Healey, David Clements. Airedale Hospitals NHS Trust, Keighley, UK

\subsection{6/gutjnl-2021-BSG.376}

Introduction Faecal elastase-1 (Fel-1) is often used for investigation of diarrhoea and if exocrine pancreatic insufficiency (EPI) is suspected ${ }^{1}$. It is common in routine clinical practice that patients with EPI have iron deficiency anaemia (IDA). Although logical that EPI patients are prone to IDA due to malabsorption, ${ }^{2,3}$ the association has not yet been shown in the published literature ${ }^{4}$. We wanted to see how strong the positive association might be between low Fel-1 (and therefore EPI) and IDA.

Methods Patients with low Fel-1 $<200 \mathrm{mcg} / \mathrm{g}$ were identified retrospectively from December 2019 to November 2020. Endoscopy, radiology, coeliac serology and ferritin results were reviewed. None of the low Fel-1 patients had an identified cancer or any other cause for IDA. Statistical analysis was performed to study the association of IDA in patients with low Fel-1.

Results 301 Fel-1 results were available over one year period 47/301 (16\%) patients were identified with low Fel-1

$26 / 47$ were females

Mean age $66(\mathrm{SD} \pm 15)$

7/47 (15\%) low Fel-1 patients had IDA and unremarkable investigations

7/7 (100\%) with low Fel-1 and IDA had diabetes or impaired glucose tolerance

$7 / 40$ (17\%) with low Fel-1 and normal ferritin had diabetes

26/254 (10\%) with normal Fel-1 had IDA

228/254 (90\%) with normal Fel-1 had normal iron

Sensitivity of finding IDA in low Fel-1 patients 0.15 (95\% CI $0.06-0.28)$

Specificity $0.90(95 \%$ CI $0.85-0.93)$

Positive predictive value $0.21(95 \%$ CI $0.09-0.39)$

Negative predictive value 0.90 (95\%CI $0.80-0.89$ )

Positive likelihood ratio 1.5 (95\%CI $0.67-3.20)$

Conclusions (1) Our study shows that low Fel-1 is positively associated with IDA.

(2) Fel-1 may have a role in investigation of unexplained iron deficiency anaemia.
(3) Fel-1 is useful in the IDA pathway especially in patients who also have diabetes

(4) A low Fel-1 may help with identifying EPI as a cause of IDA and reduce repeat cycles of endoscopic investigations by treating EPI.

\section{REFERENCES}

1. Lam KW, Leeds J. How to manage: patient with a low faecal elastase Frontline Gastroenterology 2021;12:67-73.

2. Shintakuya R, Uemura K, Murakami $Y$, et al. Sarcopenia is closely associated with pancreatic exocrine insufficiency in patients with pancreatic disease. Pancreatology. 2017 Jan-Feb;17(1):70-75. doi: 10.1016/j.pan.2016.10.005. Epub 2016 Oct 11. PMID: 27743711

3. De la Iglesia-García D, Huang W, Szatmary P, et al. Efficacy of pancreatic enzyme replacement therapy in chronic pancreatitis: systematic review and meta-analysis. Gut 2017:66:1354-1355.

4. Dominguez-Munoz, Juan Enrique, Castineira, Margarita, Larino-Noia, Jose, et al. (2010). M1391 Iron Metabolism and Iron-Deficiency Anemia in Patients with Chronic Pancreatitis. Gastroenterology. 138. 10.1016/S0016-5085(10)61815-X

\section{PTH-62 THE SAFETY AND QUALITY OF ULTRASOUND-GUIDED LIVER BIOPSY IN CLINICAL PRACTICE}

Luca Cardillo*, Momtaj Khalik, Nivian Sukirthan, Hazem Rizk, Rebecca Greenhalgh, Laith Alrubaiy. London North West University Healthcare NHS Trust, London, UK

\subsection{6/gutjnl-2021-BSG.377}

Introduction Liver biopsy constitutes an invaluable diagnostic, prognostic, decision-making and research tool in patients with liver disease. Multiple approaches and techniques are described, however evidence is scanty in many aspects. Audits evaluating complications of liver biopsies, use of different biopsy needles, biopsy size and communication between the clinician and pathologist are paramount to ensuring high-quality care. This study assesses local liver biopsy services in a non-specialised liver unit against guidelines from the British Society of Gastroenterology, the Royal College of Radiologists and the Royal College of Pathology. Findings provide a paradigm for audit and quality improvement of local liver biopsy services in other hospitals.

Methods This is a retrospective audit of electronic records. 110 ultrasound-guided percutaneous liver biopsies performed at our trust in 2019 were examined. Patients undergoing biopsy at tertiary centres were excluded. Information regarding consent, anticoagulation/antiplatelet medications, clotting and platelet levels, biopsy needles used, biopsy sample size, complications, and the pathology report were collected and anonymised. Data was processed in Microsoft Excel.

Results The current clinical practice was assessed against published guidelines. Informed consent was obtained from all patients. However, this was documented in procedural notes only in $88 \%$ of occasions. A minority of patients $(7 \%)$ were on anticoagulation/antiplatelet therapy; this was correctly withheld on $99 \%$ of procedures. INR prior to biopsy was $>1.4$ in only $1 \%$ of patients; this was adequately corrected. Platelets were checked in all patients and did not require correction. Targeted biopsies from a focal lesion correctly utilised an 18 $G$ needle in most instances. Non-targeted biopsies for diffuse parenchymal disease (DPD), however, did not utilise the recommended needle size of 16 gauge in $77 \%$ of biopsies. Indeed, DPD biopsies provided sufficient sample sizes $(>20$ $\mathrm{mm})$ in $41.2 \%$ of cases. Complications were reported in $4 \%$ of patients, and were mostly minor. Pathology reports answered the clinical question in $91 \%$ of cases, and were received within 10 days of biopsy in $83 \%$. 
Conclusions Local performance in percutaneous hepatic biopsy is safe, with low complication rates. Using the guidelines from the British Society of Gastroenterology, the Royal College of Radiologists and the Royal College of Pathology, we identified areas for improvement such as using recommended needle size and increasing liver biopsy size for diffuse parenchymal disease. These findings provide a basis for ongoing local quality improvement, and a framework on which other general hospitals can structure quality improvement whilst contributing to the evidence-basis for UK-wide guidelines.

\section{Education \& training}

\section{PTU-78 GI AND NON-GI MANIFESTATIONS OF COVID-19 DURING FIRST WAVE OF THE PANDEMIC AT DARLINGTON, UK}

\begin{abstract}
1,2Anjan Dhar*, ${ }^{2}$ Alexander Newman, ${ }^{2}$ Vikki Rand. ${ }^{2}$ County Durham and Darlington Nhs Foundation Trust, Darlington, UK; ${ }^{2}$ School of Health and Life Sciences, Teesside University, Middlesbrough, UK
\end{abstract}

\subsection{6/gutjnl-2021-BSG.378}

Anjan Dhar ${ }^{1,2}$, Alexander Newman ${ }^{2}$, Vikki Rand ${ }^{2}$

Department of Gastroenterology ${ }^{1}$, Darlington Memorial Hospital and School of Health and Life Sciences ${ }^{2}$, Teesside University, UK

Background Gastrointestinal (GI) manifestations of COVID-19 have been increasingly reported from many centres but it is not clear as to whether the presence of GI manifestations influences the outcomes of COVID-19. The data from the UK is still emerging and there is significant variability between the North of England and the rest of the UK.

Aim of this study DarCoVE was a single centre epidemiological study initiated over a 3 week period during the peak of the first wave of the COVID-19 pandemic in the United Kingdom. This prospective cohort analysis evaluated the GI and non-GI manifestations of the disease and produced a multivariate analysis of prognosticators for COVID-19.

Methods Consecutive patients admitted with fever, cough or shortness of breath to the Acute Medical Admissions Service of Darlington Memorial Hospital between 26 March 2020 12 April 2020 were recruited to an electronic database, and divided into two cohorts: RT-PCR positive for SARS-CoV-2 $(\mathrm{COVID}+)$ and negative (COVID-). Demographic parameters, underlying co-morbidities, GI and non-GI symptoms, BMI, haematological and biochemical laboratory parameters, chest radiology, need for supplemental oxygen, need for high dependency and intensive care treatment, length of hospital stay and mortality were recorded. Univariate survival analysis was performed by Cox proportional hazard model in $\mathrm{R}$, multi variate analysis was done by forward selection model, cumulative survival by Kaplan-Meier method using log-rank test.

Results 275 patients formed the dataset for analysis, 130 COVID+. Median age of COVID+ was 70(range 23-95yrs), $63 \%$ were over age $65 \mathrm{yrs}, \mathrm{M}: \mathrm{F}=1.28 .73 \%$ had at least one co-morbidity, diabetes commonest. Median BMI 29.7 (range 13.9-44.9). $60.8 \%$ patients had a $\mathrm{BMI}>30$, compared to UK average of $10.9 \%(\mathrm{p}<0.001)$. GI manifestations included: diarrhoea in $10.1 \%$, vomiting $13 \%$, abdominal discomfort $9.4 \%$, loss of appetite 5.7\%, abnormal liver functions 37\%, mean
ALT 52.4 IU/L, ALT $>150$ in 5.1\%. Of 43 clinical and biochemical factors investigated for prognostic value, 9 factors were associated with outcome at $\mathrm{p}<0.05$ with cough and diarrhoea associated with lower risk of death compared to the other 7 factors. On multivariate analysis, high frailty score $>$ 5 , worst oxygenation $\mathrm{SpO}_{2}<93 \%$, platelets $<100 \times 10^{9} / \mathrm{L}$ and immunocompromised were poor prognosticators. None of the GI manifestations co-related with risk of death in this analysis, with a trend for ALT $>150$ to be associated with higher mortality. Overall mortality was $30.8 \%$ compared to UK national mortality of $26 \%$, with ITU mortality higher at $37 \%$.

Conclusion This study has shown a regional variation in the outcome of COVID-19, with slightly different prognosticators. GI manifestations continue to be significant in COVID-19, with a trend seen with high ALT. The data from this analysis will help management in future pandemics.

\section{PTU-79 DEVELOPMENT OF A VIRTUAL REALITY TRAINING CURRICULUM FOR ERCP}

${ }^{1,2}$ Yousef Khouli*, 1,2 Karim Belhaj, ${ }^{3}$ Jad Khoury, ${ }^{1,2}$ Bijendra Patel. 'Barts Cancer Institute, London, UK; ${ }^{2}$ Queen Mary University of London, London, UK; ${ }^{3}$ University Hospitals of North Midlands NHS Trust, Stoke-on-Trent, UK

\subsection{6/gutjnl-2021-BSG.379}

Introduction Assessment of Endoscopic skills involved in performing Endoscopic Retrograde Cholangiopancreatography (ERCP) in procedural environment is complex. Tutors and experts emphasize the need to develop and use precise and significant assessments measuring tools that are valid for evaluating trainees' progress in obtaining essential gastroenterologyrelated procedural skills. The purpose of this research was to develop a structured evidence-based virtual reality training curriculum and set a proficiency performance benchmark for a set of objective metrics of endoscopic skills during ERCP procedures using the Simbionix GI Mentor 2 Simulator. Our second aim of the study was to form a face and construct validity of the simulator for the ERCP module to show significant differences between competent and non-competent operators in that module.

Keywords: Simbionix, GI Mentor 2, ERCP Module, ERCP training, Simulation, ERCP simulators, Benchmark, Virtual Reality, Training curriculum

Materials and Methods In this study, a total of 39 participants were divided into three groups according to their level of experience and number of endoscopies performed in their career (Novices, Intermediate, and Experts). They were required to perform ERCP procedure, case number 2 in Module 1 in the GI Mentor 2 Simulator, and upon completion of the study, they were asked to fill a questionnaire about the simulator, simulation in general, and their previous experience. The time taken for task completion, number of papillary contacts before cannulation, number of cannulation to the Pancreatic Duct, and other metrics calculated from the simulator along with the questionnaire results were collected and compared between the groups. The first group consisted of novices; medical students, foundation doctors, and core trainees, with no previous knowledge or exposure to ERCP or endoscopic procedures. 